\title{
Wafer scale fabrication of graphene microelectrode arrays for the detection of DNA hybridization
}

\author{
R. Campos ${ }^{1}$, G. Machado Jr. ${ }^{1,2}$, M.F. Cerqueira ${ }^{2}$, J. Borme ${ }^{1}$, P. Alpuim ${ }^{1,2^{*}}$ \\ 1 - International Iberian Nanotechnology Laboratory (INL) 4715-330, Braga, Portugal \\ 2 - Department of Physics, University of Minho, 4710-057, Braga, Portugal \\ *pedro.alpuim.us@inl.int
}

\section{Abstract}

Since the discovery of graphene, and due to its unique properties, we have witnessed a growing interest in the use of graphene-based devices for applications in the most diverse areas, namely in biosensing, particularly in the detection of genetic material. DNA can bind directly to graphene without the need of a linker and that makes this platform highly interesting for biosensor development.

Here, electrochemical chips consisting of 6 independent gold microelectrode arrays as working electrode, and platinum reference and counter electrodes were fabricated at the wafer scale and, after graphene transfer and patterning, were used in the detection of DNA hybridization. Combining the sensitivity of electrochemical impedance spectroscopy and the selectivity of DNA beacons, we were able to detect DNA hybridization in a linear range between $5 \mathrm{pM}$ and $5 \mathrm{nM}$, which is in the relevant clinical range for many diseases, with sensitivity to single nucleotide polymorphism.

\section{Graphical abstract}

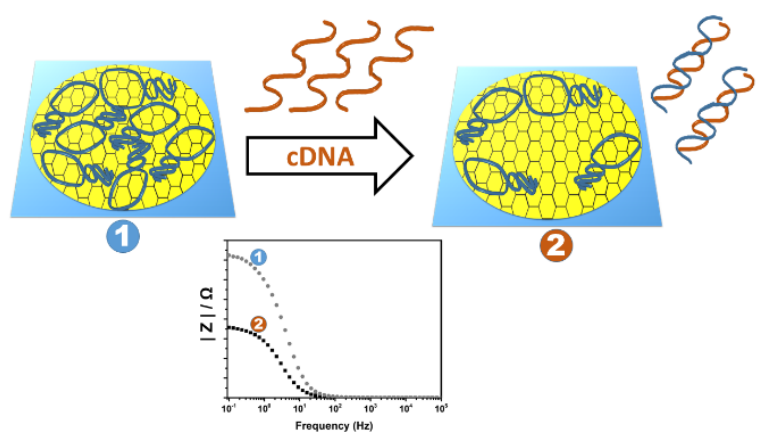

\section{Keywords}

Graphene; genosensor; electrochemical chip; single nucleotide polymorphism

\section{Introduction}

The medical community needs detection systems that are fast, reliable and easy to use. DNA biosensors are a technology that is growing rapidly and that presents itself as an alternative to the classical gene assay.[1-4] Apart from the low-cost, simplicity and miniaturization possibility, the ability to detect single nucleotide polymorphism is one of the main advantages that DNA biosensor technologies present. Single nucleotide polymorphism is a variation in a single nucleotide that occurs at a specific position in the genome, and has been correlated with various disorders/diseases, including cystic fibrosis, Alzheimer's, Parkinson's, diabetes and various cancers.[5-8] 
2D materials are currently used to help lower the limits of detection and to improve the performance of the biosensors. Their relevance stems from their specific electronic, optical and mechanical properties.[9,10] Among 2D materials, graphene is the one to which most of the attention has been dedicated so far. The sheet of sp2-bounded carbon atoms arranged in a honeycomb lattice proves to be in several respects an ideal platform for biosensing, since it provides a chemically and mechanically stable surface for interfacing with molecules, with an exposed 2D electron gas with high carrier mobility. Moreover carrier concentration can be tuned in graphene by simple application of a gate voltage.[11,12]

The adsorption of DNA on graphene, or its derivatives such as graphene oxide (GO) or reduced graphene oxide (rGO), has been used for biosensing applications since 2009, when Chen and co-workers [13] used a fluorescent labeled DNA strand to detect target DNA and proteins. They showed that when the dye-labeled single strand DNA (sSDNA) is bound to the GO surface the fluorescence is quenched and that after interaction of the ssDNA with target DNA or with proteins the ssDNA DNA is released from the GO surface and the fluorescence is restored. Bonanni and Pumera[14] also explored the interaction between DNA and graphene to developed an impedimetric genosensor that could detect the SNP associated with Alzheimer's disease. When the probe DNA is deposited on the graphene surface its bases bind via $\pi-\pi$ interactions, and then when target DNA (cDNA) is added there are three possible mechanisms for the formation of the duplex: i) the Langmuir-Hinshelwood model: the cDNA adsorbs on the graphene surface, diffuses and forms duplex, which then leaves the surface; ii) the Eley-Rideal model: the duplex is formed directly on the surface without cDNA adsorption, and iii) the displacement model: the probe DNA is displaced from the surface by cDNA into solution and then reacts with another cDNA in solution.[12]

Microelectrode arrays besides possessing all the advantages of the microelectrodes - higher current density, faster mass transport and lower detection limits - allow signal amplification due geometry.[15,16] Due to these advantages microelectrode arrays create the opportunity for lab-on-a-chip devices and in-vitro and in-vivo measurements. Microelectrode arrays can be fabricated using i) assembly techniques, ii) screen-printing techniques, iii) electrodeposition (also known as electroplating) and, iv) photolithography. Microelectrodes fabricated using assembly techniques include those fabricated using assembly of materials or molecules. The assembly of materials is a physical technique which can be performed by an unskilled laboratory worker, uses materials that are readily available (can be metals: such as gold, platinum, silver and others or carbon in the form of fiber, paste, graphite or glassy carbon) and does not required special laboratory equipment.[15,16] Molecular assembly includes those methods in which a mixed self-assembled monolayer is deposited and then one of the components is selectively desorbed from the surface, thus creating the microelectrodes.[17,18]

The second method for fabrication of microelectrodes is the screen printing technique. It allows the mass production of identical electrodes, by using simple machinery and operation in order to screen-print both the conductors and the insulators, with the desired pattern. The inks used for the printing are based on a suspension of conducive materials. Like in the case of the assembly technique the most common compounds used for the conductors are either metal particles or carbon based materials.[16,19]

Electrodeposition is a process that uses electrical current to reduce cations of a desired material from a solution and coat that material as a thin film onto a conductive substrate surface. Electrodeposition, including patterned electrodeposition (electrodeposition performed on a designed array support) presents itself as a very promising alternative microelectrode fabrication technique, especially for the fabrication of nanostructures, and has received an increasing interest in the recent literature.[20,21] Wang et al.[21] proposed a method for the co-deposition of PEDOT (poly(3,4-ethylene-dioxythiophene)) and graphene on microelectrodes which led to a biosensor that presents lower impedance and better local field potentials

Among the techniques of microelectrode array fabrication photolithography is the most complex but is also among those yielding smallest feature sizes, while retaining the ability to reliably produce a high number of identical arrays at the same time[15,16]. However, photolithography requires access to a clean room which limits its use to cases where the benefits brought by the feature size compensate the higher cost barrier.[15,16] 
In order to fabricate high quality microelectrode arrays by photolithography there are three steps that are crucial, i) photo mask design, ii) photoresist spinning and exposure and, iii) photoresist development. The photo mask is a nearly optical flat glass or a quartz plate covered in chromium and a photoresist. The photomask design is related to the writing of a specific pattern on a mask into a layer of photoresist using a direct write laser. The pattern is transferred from the photoresist into the chromium by chemical etching. The photomask can be placed in direct contact with the production wafer coated with photoresist while UV light is shined trough the mask. A reduced image of the photomask can also be projected into the photoresist of the target wafer. For applications that do not require a high throughput, during the optimization of the design and of the fabrication process, the fabrication of the photomask can be replaced by a direct write on the target wafer using a laser. Adequate choice of photoresist and optimization of the process conditions allow the creation of sharp vertical photoresist walls, which in turn leads to the accurate transfer of the photoresist pattern into the materials deposited on the wafer, which is among its main advantages.[15]

Here we fabricated arrays of microelectrodes of $40 \mu \mathrm{m}$ of individual size, by employing direct-write laser photolithography to define the pattern in a gold thin film followed by the definition of additional electrodes and passivation layers. The fabricated arrays were covered with a graphene monolayer in order to allow functionalization with DNA. The resulting sensor was able to detect concentrations of fully complementary DNA in the range between $5 \mathrm{pM}$ to $5 \mathrm{nM}$, while keeping the sensitivity to single nucleotide polymorphism.

\section{Experimental}

Materials. All components of buffer solutions $\left(\mathrm{Na}_{2} \mathrm{HPO}_{4}, \mathrm{NaH}_{2} \mathrm{PO}_{4}, \mathrm{NaCl}\right.$ and $\left.\mathrm{MgCl}_{2}\right)$ and all the solvents were from Sigma-Aldrich. Ultrapure water (18 $\mathrm{M} \Omega \mathrm{cm}$, Millipore, Bedford, MA, USA) was used throughout the experiments. The probe DNA, (5'- AGC TTC ATA ACC GGC GAA AGG CTG AAG CT-3') had a C7amino modification on the 3' end and a Atto MB2 modification on the 5', the complementary DNA (5'-AGC TTC AGC CTT TCG CCG GTT ATG A-3') and the SNP containing target (5'-AGC TTC AGC CTT $\underline{\text { ACG }}$ CCG GTT ATG A-3') were synthesized by Metabion International AG, Martinsried, Germany. The melting temperature, $T_{\mathrm{m}}$, of the DNA was estimated using DINAMelt web server[22], and, in the hybridization buffer (10 mM PB/150 mM NaCl/50 mM MgCl 2 ) was $63.9 \stackrel{\circ}{\circ}$ for the probe DNA (beacon), $82.7 \stackrel{\circ}{\mathrm{C}}$ for the fully complementary duplex, and $80.8 \stackrel{\circ}{\circ}$ for the single nucleotide polymorphism containing duplex, while in the working buffer (10 mM PB) it was, respectively, $42.9^{\circ} \mathrm{C}, .58 .9{ }^{\circ} \mathrm{C}$ and $55.1^{\circ} \mathrm{C}$.

The high purity (> 99.99\%) $25 \mu \mathrm{m}$ coper foils used for the graphene growth were purchased from AlfaAesar and Goodfellow.

Design of the microelectrode arrays. Each array is composed of 60 disks of $40 \mu \mathrm{m}$ arranged in 11 lines of 5 or 6 , forming a triangular lattice with a spacing of $400 \mu \mathrm{m}$ (figure 1B) in order to avoid overlapping of the diffusion layers[23], and connected between them by lines of $12 \mu \mathrm{m}$ of width forming a planar truss. Two larger additional contacts are present (figure $1 \mathrm{~A}$ ), $11.4 \mathrm{~mm} \times 3.7 \mathrm{~mm}$, used as counter and reference electrodes. The electrical contacts are driven to the edge of the chip, to electricals pads with $1 \mathrm{~mm}$ pitch. The chip is designed to measure $21.75 \mathrm{~mm} \times 21.75 \mathrm{~mm}$ after dicing, mating an edge card connector (Samtec MB1-120 for $0.80 \mathrm{~mm}$ thick board) connected to the measurement system.

Fabrication of the pre-patterned microelectrode arrays. The substrate used is a $200 \mathrm{~mm}$ Silicon wafer (B-doped, 8-30 $\Omega \cdot \mathrm{cm}$, LG Siltron) with $200 \mathrm{~nm}$ of thermal oxide. The wafer is sputtered with $\mathrm{Cr} 3 \mathrm{~nm}$ / Au $40 \mathrm{~nm}$, where the chromium acts as an adhesion layer. An optical lithography using direct write layer (photoresist AZ1505, $1035 \mathrm{~nm}$ ) defines and protects the contacts: disks, current lines and additional electrodes. The contacts are etched by ion milling using an incident flow of neutralized argon ions at $40^{\circ}$ from normal incidence using mass spectrometer of the residual gases to determine the end of the process. 


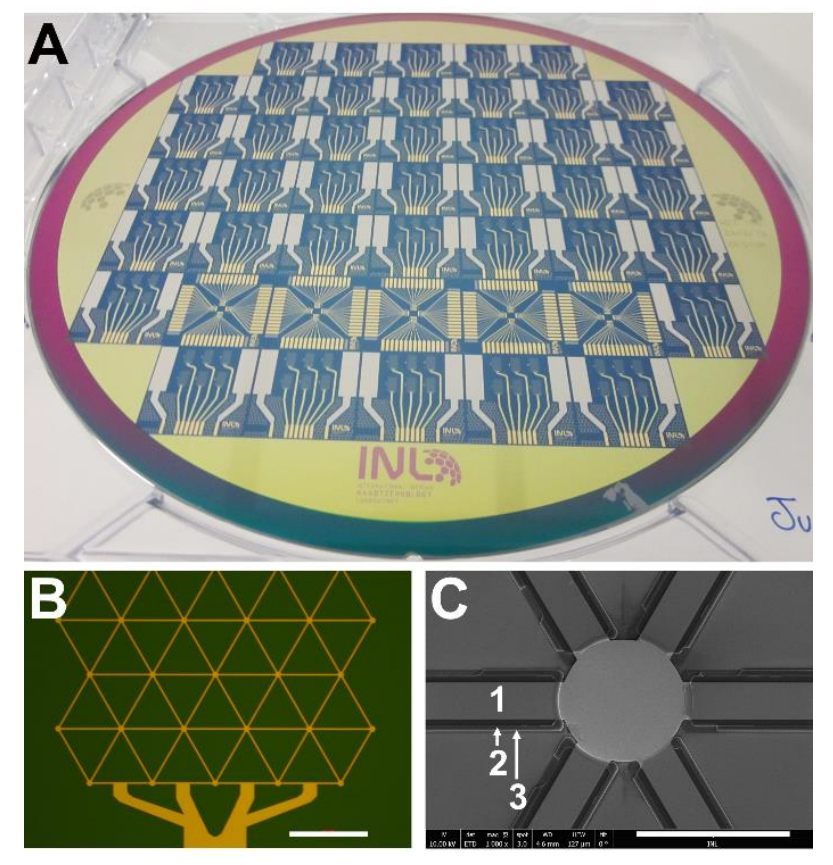

Figure 1-A) Optical image of the $200 \mathrm{~mm}$ full wafer with the electrochemical chips. In each chip two large contacts to be used as reference and counter-electrodes, respectively, are visible. B) Detail of one of the arrays, showing the arrangement of the micro-electrodes in an array and the connection lines, after ion milling of the gold. - working electrode. C) SEM image of a single microelectrode. Six lines connect the electrode to its neighbors. The gold lines are covered with a stack of silicon nitride and oxide, noted 1). The sides of the passivated gold layer, which is composed of passivation stack on the oxidized silicon, is noted 2). The outer passivation, which is suspended due to the removal of the stopping layer underneath, is noted 3). Figure $\mathrm{S} 1 \mathrm{in} \mathrm{ESI}$ is a full size image where 1,2 and 3 are easily noted. The scale bar in B is $500 \mu \mathrm{m}$ and in $\mathrm{C}$ is $50 \mu \mathrm{m}$.

Next, an insulation of the electrical lines is done. To this end, an optical lithography is performed, clearing photoresist on the lines to be insulated. A stopping layer with composition $\mathrm{Al}_{2} \mathrm{O}_{3} 10 \mathrm{~nm} / \mathrm{TiWN} 15 \mathrm{~nm} /$ AlSiCu $100 \mathrm{~nm} /$ TiWN $15 \mathrm{~nm}$ is sputtered onto the wafer, and lift-off is performed in a stripper (Fujifilm Microstrip 3001) at $65^{\circ} \mathrm{C}$ with ultrasonic agitation. This stopping layer covers the gold electrodes and the silicon oxide, protecting them from the reactive ion etching process of the passivation layer. It starts $2 \mu \mathrm{m}$ away from the current lines, because the current lines and the area within $2 \mu \mathrm{m}$ will be covered in the passivation. The wafer is then covered with the passivation multilayer grown by plasma-enhanced CVD and composed of [SiN $\left.50 \mathrm{~nm} / \mathrm{SiO}_{2} 50 \mathrm{~nm}\right]_{2} / \mathrm{SiN}_{x} 50 \mathrm{~nm}$. An optical lithography defines the area to be passivated. Reactive ion etching (RIE) is used to etch the multilayer. During this process, the top layer of TiWN is etched away, and the process of etching stops on the aluminium layer. The remaining photoresist is stripped by oxygen plasma and the stopping layer is removed by wet etch. First, the aluminium is removed by brief exposure to the photoresist developer (AZ400K 1:4). Then, the TiWN layer is removed by dissolution in $30 \%$ hydrogen peroxide. Finally, $\mathrm{Al}_{2} \mathrm{O}_{3} 10 \mathrm{~nm}$ is removed by exposure to $4 \mathrm{~min}$ in the photoresist developer. It is to be noted that the alumina layer acts as a diffusion barrier, preventing the diffusion of aluminium from the stopping layer into gold (which would create a less conductive intermetallic). The TiWN prevents the intermixing of aluminium and alumina, creating phases more difficult to remove by the soft wet etch used. This combination of protection layer remains easy to be removed without letting detectable contamination on gold. The passivation layer extends $4 \mu \mathrm{m}$ beyond the width of the current line, overlapping the limit of the stopping layer. This overlap is necessary to make sure that no oxide is left exposed to the reactive ion etching, which would result in opening an access to the doped silicon and a possible short circuit. After the removal of the stopping layer by wet etch, the passivation layer is left suspended in the outer $2 \mu \mathrm{m}$. The suspended part can easily break during processes using ultrasonic 
agitation. This effect can be seen on figure 1C. The broken insulator in the outer $2 \mu \mathrm{m}$ of overlap does not cause any malfunction of the device.

The next lithography is used to open the area of the electrode used as a reference. A layer of platinum $20 \mathrm{~nm}$ is sputtered onto the exposed gold area of this electrode. A lift-off is performed in acetone in ultrasonic agitation. Another optical lithography is used to open the areas of the pads, then TiWN $50 \mathrm{~nm}$ is deposited and lifted-off in Microstrip 3001. This strong and conductive nitride protects the gold underneath to allow multiple mating of the silicon card with the connector. The wafer is then sputtered with $\mathrm{Al}_{2} \mathrm{O}_{3} 10 \mathrm{~nm}$ and an optical lithography open the disks where graphene is going to be transferred. A wet etch using 4 min exposure to the photoresist developer removes the $\mathrm{Al}_{2} \mathrm{O}_{3}$ from inside the disk. The purpose of the $\mathrm{Al}_{2} \mathrm{O}_{3}$ layer is to protect the exposed gold of the planar gate from a future exposure to oxygen plasma, which will etch around the gold/graphene disks and might cause damage to the thin film exposed gold. After this step, the pre-patterned wafer is ready to be used for graphene transfer.

Graphene growth and transfer. Single-layer graphene is grown on copper catalyst by Chemical Vapour Deposition (CVD) in a three-zone quartz furnace (FirstNano EasyTube 3000). The $100 \mathrm{~mm} \times 100$ $\mathrm{mm}$ copper foil is placed inside a graphite confinement box, which reduces the gas flow arriving on the catalyst and the contamination in silicon coming from the walls of the oven. Then the copper foil is annealed at $1020^{\circ} \mathrm{C}$ for $20 \mathrm{~min}$ in $0.5 \mathrm{mTorr}$ hydrogen atmosphere. These conditions are kept constant while a gaseous mixture $\mathrm{H}_{2}: \mathrm{CH}_{4} 6: 1$ is introduced in the hot furnace for 30 min for graphene growth.

The graphene is transferred onto the contacts using the temporary polymeric (PMMA) substrate method. After graphene growth, the quality of the graphene layer is assessed by Raman spectroscopy on both sides of the copper foil. The side with the better quality is spun with $600 \mathrm{~nm}$ of PMMA 950k. The opposite side is exposed to an oxygen plasma $\left(\mathrm{O}_{2}: \mathrm{Ar} 2: 1,0.9 \mathrm{mTorr}, 250 \mathrm{~W}, 2 \mathrm{~min}\right)$ in order to remove graphene. The copper foil is then dipped into an aqueous solution of $\mathrm{FeCl}_{3} 0.5 \mathrm{M}$ at $35^{\circ} \mathrm{C}$, for $30 \mathrm{~min}$. Copper is dissolved, leaving the floating PMMA layer as the substrate of graphene. The PMMA/graphene layer is cleaned in a $2 \%$ solution of hydrochloric acid for $30 \mathrm{~min}$ to dissolve the Fe precipitates. It is further cleaned in deionized water (resistivity $\geq 18 \mathrm{M} \Omega \cdot \mathrm{cm}$ ) for $5 \mathrm{~min}$. The cleaning steps in $\mathrm{HCl}$ and water are repeated 5 times. The PMMA/graphene layer is transferred onto a silicon substrate with pre-patterned microelectrode arrays. Before the transfer, the pre-patterned contacts are exposed to a vapour HDMS priming (Yield Engineering Systems) at $150{ }^{\circ} \mathrm{C}$ and 5.9 Torr for $5 \mathrm{~min}$ in order to turn it more hydrophobic and ease the removal of water lying between the pre-patterned contacts and graphene. Graphene, which lies in the bottom side of the PMMA layer, is forced onto the electrical contacts by using a gentle nitrogen blow from the top. The remaining water is removed by annealing at $180^{\circ} \mathrm{C}$ following a 1 hour ramp from ambient temperature, and keeping the higher temperature for 12 hours. The PMMA is dissolved in acetone bath for 2 hours. The transfer process is repeated for different pieces of graphene until the whole area of the wafer is covered with graphene.

Graphene patterning. After transfer, a final optical lithography defines and protects the graphene disk with $1035 \mathrm{~nm}$ photoresist. An exposure to the oxygen plasma (same conditions as above, $2 \mathrm{~min}$ ) to etch graphene is then performed. The process is calibrated by the duration to remove $100 \mathrm{~nm}$ of photoresist together with the single layer graphene (SGL), as confirmed by mechanical profilometry. After this, acetone is used to remove the remaining photoresist and another photoresist coating is prepared for the wafer dicing step (Disco DAD 3350). During the dicing process, the water flow cooling the blade is reduced by half as compared to standard process in order to prevent that the strong pressure created by the incident water flow on the wafer lifts graphene away. After dicing, samples are cleaned with acetone to remove photoresist (without ultrasonic agitation) and ethylacetate, which dissolves any remaining PMMA, and finally dried by a nitrogen blow.

DNA sensor. After the graphene patterning the probe DNA was immobilized on the graphene surface ( $10 \mu \mathrm{L}$ drop with a concentration of $10 \mu \mathrm{M}$ in hybridization buffer) and allowed to react for 2 hours. The aromatic groups of the DNA bases bind to the graphene surface via $\pi-\pi$ interactions.[24] After that the chip was gently rinsed with the hybridization buffer, in order to remove weakly attached strands, and 
characterized electrochemically (cyclic voltammetry and electrochemical impedance spectroscopy) using a solution containing $1 \mathrm{mM}$ of $\mathrm{K}_{3} \mathrm{Fe}(\mathrm{CN})_{6}$ and $1 \mathrm{mM}$ of $\mathrm{K}_{4} \mathrm{Fe}(\mathrm{CN})_{6}$ and the working buffer as electrolyte. An Autolab PGSTAT302N running NOVA 2.1 was used for the electrochemical measurements.

\section{Results}

Graphene microelectrode arrays. Figure 1A shows an optical image of a $200 \mathrm{~mm}$ wafer patterned with the electrochemical chips. In figure 1B a zoomed-in view of one microelectrode array is displayed, and figure $1 \mathrm{C}$ shows a scanning electron microscopy (SEM) image of an individual microelectrode. It is possible to see in figure 1B not only individual microelectrodes within the array but also the "arm" that connects the array to the conduction lines and pads. Figure $1 \mathrm{C}$ shows the individual disk electrode and the six contact lines that connect it to its nearest neighbors within the array. The gold interconnects are covered with a passivation multilayer which extends an additional $4 \mu \mathrm{m}$ to each side of the metal line (see section 2).

The electrochemical characterization of the fabricated chips, without graphene, i.e. with bare gold electrodes, is shown in figure 2. The electrochemical area was determined by recording cyclic voltammograms using $\mathrm{H}_{2} \mathrm{SO}_{4}$. The obtained result is shown in figure $2 \mathrm{~A}$, with an estimated electrochemical area of $1.2 \times 10^{-3} \mathrm{~cm}^{2}$, determined using the oxide reduction peak.[25] This area is only 1.5 larger than the geometrical area $\left(7.5 \times 10^{-4} \mathrm{~cm}^{2}\right)$ owing to the smooth gold surface obtained by sputtering. This small
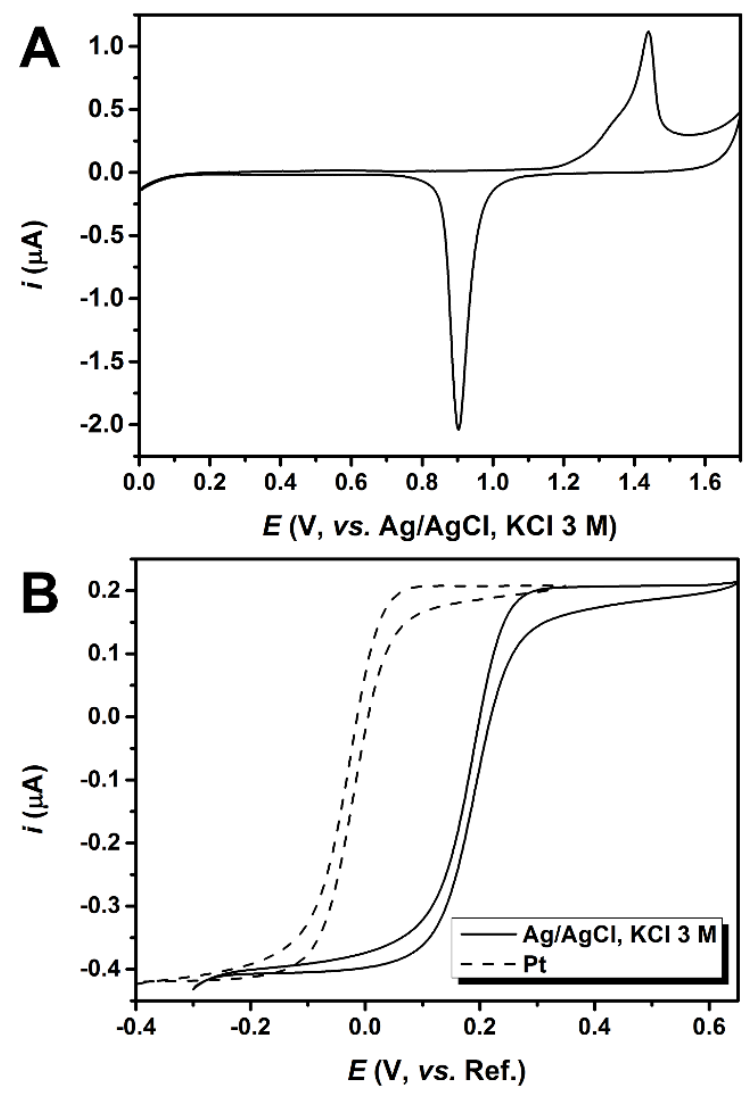

Figure 2 - Electrochemical characterisation of the fabricated electrochemical chips. The chips used here did not have graphene transferred on them so that we could evaluate the electrochemical behaviour of the fabricated devices. In A) is shown a cyclic voltammogram (scan rate $=300 \mathrm{mV} \mathrm{s}^{-1}$ ) of one array in a solution of $\mathrm{H}_{2} \mathrm{SO}_{4} 0.1 \mathrm{M}$; the electrochemical area can be determined from the reduction peak. B) shows that the in-chip pseudo-reference electrode works as expected since there is no difference in the CV shape or intensity, apart from the expected shift in $\mathrm{E}_{1 / 2}$. The working solution was $1 \mathrm{mM} \mathrm{K}_{3} \mathrm{Fe}(\mathrm{CN})_{6}, 1 \mathrm{mM} \mathrm{K} \mathrm{K}_{4} \mathrm{Fe}(\mathrm{CN})_{6}$ and $100 \mathrm{mM} \mathrm{KNO}_{3}$ as electrolyte. 
roughness factor (real area divided by the geometrical area) contributes to the success of the graphene transfer.[26]

To test our in-chip reference and counter electrodes an electrochemical characterization was performed in a $2 \mathrm{mM}$ equimolar solution of ferro/ferricyanide. The results, shown in figure $2 \mathrm{~B}$, indicate that only the half-wave potential $\left(E_{1 / 2}\right)$ changes when moving from a standard reference electrode $(\mathrm{Ag} / \mathrm{AgCl}, \mathrm{KCl} 3 \mathrm{M})$ and external $1 \mathrm{~cm}^{2} \mathrm{Pt}$ flag as counter electrode, to our in-chip $42 \mathrm{~mm}^{2}$ reference and counter Pt electrodes. This result indicates that our in-chip pseudo-reference electrode works as expected since there is no difference in the CV shape or intensity.

After graphene transfer and patterning, the electrodes were characterized using confocal Raman spectroscopy. The Raman scattering experiments were performed at room temperature in a back scattering geometry on an Alpha300 R confocal Raman microscope (WITec). The system was operated with an excitation laser power of $1 \mathrm{~mW}$ using a $532 \mathrm{~nm}$ laser line. Figure $3 \mathrm{~A}$ shows a Raman map of the graphene electrode area. The image was obtained with the Wltec clustering tool which compares the user-selected range of $D, G$ and $2 D$ modes in the graphene Raman spectra stored in each image pixel with the same ranges of the 3-dimensional Raman spectra basis showed in figure 3B. To build the image, each pixel was attributed the color of the particular base spectrum (figure 3B) that most resembled the Raman spectrum stored in that pixel, in the chosen range. The measurements were performed in an area of $300 \times 300 \mu \mathrm{m}^{2}$, resolved into $100 \times 100$ Raman spectra.
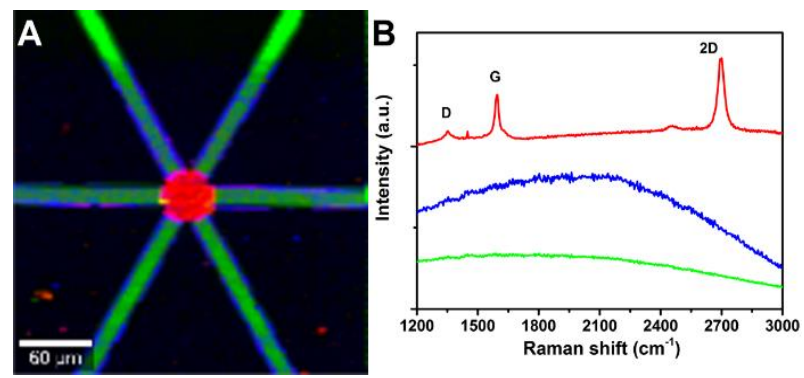

Figure 3- Raman map (A) and spectra (B) of one microelectrode area after graphene transfer. It can be seen that the graphene only covers the microelectrode, and not the interconnects or surrounding areas, following the lithographic mask. The scale bar in $A$ is $60 \mu \mathrm{m}$.

From Figure 3 it can be concluded that the graphene on the sensor/microelectrode is of good quality and corresponds to monolayer graphene (the $\mathrm{I}_{2 \mathrm{D}} / \mathrm{I}_{\mathrm{G}}>>1$ and the $\mathrm{FWHM}_{2 \mathrm{D}} \approx 35 \mathrm{~cm}^{-1}$ ). This Raman study reveals that graphene only covers the electrodes area, as desired and designed in the lithographic process.

Figure 4 shows the Bode plots for the various stages of the biosensor development (Nyquist and cyclic voltammetry plots are shown in figures S2 and S3 of ESI, respectively). Starting from the bare electrode there is a tenfold increase in charge transfer resistance ( $\left.R_{c t}\right)$ when $10 \mu \mathrm{L}$ of $10 \mu \mathrm{M}$ probe DNA (ssDNA) are allowed to interact with the graphene surface for 2 hours. This increase in $R_{c t}$ is expected since the probe 
DNA binds to the graphene surface via electrostatic interactions and the negatively charged phosphate groups of the DNA backbone will repel the also negatively charged redox couple.

The probe DNA modified sensor is then exposed to different concentrations of target DNA and, as the concentration of target DNA increases (from $5 \mathrm{pM}$ to $50 \mathrm{nM}$ ) the impedance of the array decreases due to fact that more uncovered graphene surface is available for electrochemical reaction. It is known that the ssDNA bounds to the graphene surface via $\pi-\pi$ interactions. However, after reaction with the complementary DNA, the part of the DNA duplex that is available for chemical interaction are the phosphate groups which do not bind to the graphene surface. Therefore, after hybridization the DNA duplex leaves the surface which becomes available for electrochemical reaction.[27]
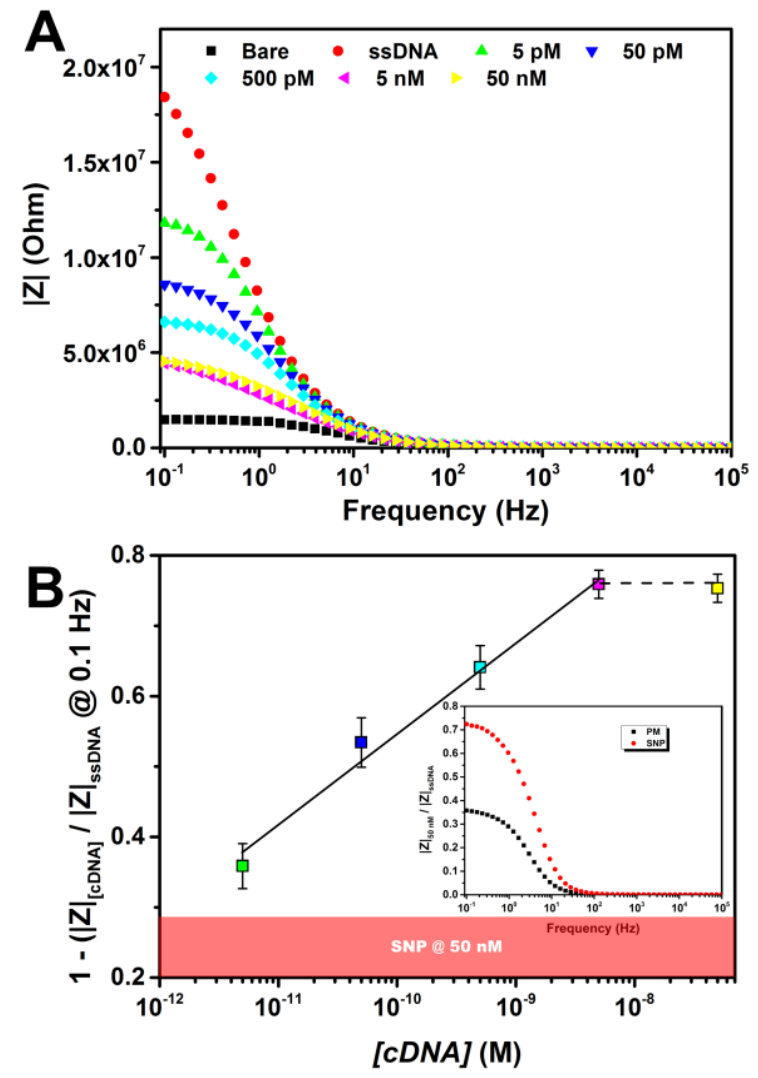

Figure 4 - A) Bode plots for the different experimental conditions, from bare electrode to $50 \mathrm{nM}$ of complementary DNA. B) The calibration curve for the sensor extracted from Bode plots in A). The red area corresponds to the \% of signal variation for the highest concentration of CDNA with SNP. The inset shows normalised Bode plots showing the sensor's ability to detect SNP.

Using a similar approach, Bonanni and Pumera [14] observed that, after immobilization of probe DNA on graphene platforms, the reaction with fully complementary target DNA resulted in a decrease of $R_{c t}$, while in the case of SNP containing target DNA the decrease in $R_{c t}$ was much smaller, and negligible in the case of non-complementary DNA. Following a similar trend we show here a linear range that includes four orders of magnitude (from $10^{-12}$ to $10^{-9} \mathrm{M}$ ), and a sensitivity of $2.4 \times 10^{6} \mathrm{\Omega} /$ decade (Figure $4 \mathrm{~A}$ ). Figure 4B shows a calibration curve for the sensor based on the measurements of Figure 4A (Bode plots). Since the variation in $R_{c t}$ between ssDNA and the lowest concentration of cDNA studied here is $35 \%$, it should be possible to build a sensor that can go to lower detection limits than those presented here. The inset in Figure 4B shows the sensors ability to detect SNP. Even at the highest SNP concentration the change in signal after reaction of SSDNA with SNP containing cDNA is less than $30 \%$, which is lower than that of the lowest concentration of perfect match cDNA tested. Therefore the limit of detection of this sensor can be 
considered to be $5 \mathrm{pM}$ because this is the lowest concentration that we can differentiate from the control measurement, which in this case is not a blank but the SNP measurement. Even though the sensor seems to be able to detect lower concentrations we did not attempt that in the present study.

\section{Conclusions}

Here we fabricated electrochemical chips that consisted of platinum reference and counter electrodes and six independent graphene microelectrode arrays that function as working electrode. The graphene microelectrode arrays were made by transferring and patterning graphene grown by CVD onto the fabricated gold microelectrode arrays. The characterization of the fabricated chips was made using cyclic voltammetry and electrochemical impedance spectroscopy.

It is shown that the graphene microelectrode array can detect DNA hybridization down to $5 \mathrm{pM}$, with a linear range up to $5 \mathrm{nM}$ and a sensitivity of $2.4 \times 10^{6} \Omega$ /decade. The ability of the biosensor to SNP discrimination is shown using $50 \mathrm{nM}$ of SNP containing cDNA.

\section{Acknowledgements}

This work was partially supported by the Portuguese Foundation for Science and Technology (FCT) in the framework of the Strategic Funding UID/FIS/04650/2013. G. Machado Jr acknowledges a PhD grant (no. 237630/2012-5) from CNPq-Brazil.

\section{References}

[1] J. Wang, Electrochemical biosensors: Towards point-of-care cancer diagnostics, in: Biosens. Bioelectron., 2006: pp. 1887-1892. doi:10.1016/j.bios.2005.10.027.

[2] R.Y. Lai, Folding- and Dynamics-Based Electrochemical DNA Sensors, in: Methods Enzymol., 2017: pp. 221-252. doi:10.1016/bs.mie.2017.02.001.

[3] S.S. Mahshid, S. Camiré, F. Ricci, A. Vallée-Bélisle, A Highly Selective Electrochemical DNA-Based Sensor That Employs Steric Hindrance Effects to Detect Proteins Directly in Whole Blood, J. Am. Chem. Soc. 137 (2015) 15596-15599. doi:10.1021/jacs.5b04942.

[4] S.S. Mahshid, F. Ricci, S.O. Kelley, A. Vallée-Bélisle, Electrochemical DNA-Based Immunoassay That Employs Steric Hindrance to Detect Small Molecules Directly in Whole Blood, ACS Sensors. 2 (2017) 718723. doi:10.1021/acssensors.7b00176.

[5] J. Roy, B. Mallick, Altered gene expression in late-onset Alzheimer's disease due to SNPs within 3'UTR microRNA response elements, Genomics. 109 (2017) 177-185. doi:10.1016/j.ygeno.2017.02.006.

[6] J.C. Pezzi, C.M.B.E. de Bem, T.J. da Rocha, A.F. Schumacher-Schuh, M.L.F. Chaves, C.R. Rieder, M.H. Hutz, M. Fiegenbaum, A.L. Camozzato, Association between DNA methyltransferase gene polymorphism and Parkinson's disease, Neurosci. Lett. 639 (2017) 146-150. doi:10.1016/j.neulet.2016.12.058.

[7] R.A. Hubner, R.S. Houlston, Single Nucleotide Polymorphisms and Cancer Susceptibility, in: W.B. Coleman, G.J. Tsongalis (Eds.), Mol. Basis Hum. Cancer, Springer New York, New York, NY, 2017: pp. 231-239. doi:10.1007/978-1-59745-458-2_14.

[8] B.P. Hull, P. Jiramongkolchai, J.H. Turner, L. Olson, R.K. Chandra, Single nucleotide polymorphisms related to cystic fibrosis in chronic rhinositus-a pilot study, Int. Forum Allergy Rhinol. 7 (2017) 467-473. 
doi:10.1002/alr.21926.

[9] R. Kurapati, K. Kostarelos, M. Prato, A. Bianco, Biomedical Uses for 2D Materials Beyond Graphene: Current Advances and Challenges Ahead, Adv. Mater. 28 (2016) 6052-6074. doi:10.1002/adma.201506306.

[10] C. Zhu, D. Du, Y. Lin, Graphene-like 2D nanomaterial-based biointerfaces for biosensing applications, Biosens. Bioelectron. 89 (2017) 43-55. doi:10.1016/j.bios.2016.06.045.

[11] M. Kim, J. Jang, C. Cha, Carbon nanomaterials as versatile platforms for theranostic applications, Drug Discov. Today. 22 (2017) 1430-1437. doi:10.1016/j.drudis.2017.05.004.

[12] B. Liu, S. Salgado, V. Maheshwari, J. Liu, DNA adsorbed on graphene and graphene oxide: Fundamental interactions, desorption and applications, Curr. Opin. Colloid Interface Sci. 26 (2016) 41-49. doi:10.1016/j.cocis.2016.09.001.

[13] C.H. Lu, H.H. Yang, C.L. Zhu, X. Chen, G.N. Chen, A graphene platform for sensing biomolecules, Angew. Chemie - Int. Ed. 48 (2009) 4785-4787. doi:10.1002/anie.200901479.

[14] A. Bonanni, M. Pumera, Graphene Platform for Hairpin-DNA-Based Impedimetric Genosensing, ACS Nano. 5 (2011) 2356-2361. doi:10.1021/nn200091p.

[15] S. Szunerits, L. Thouin, Microelectrode Arrays, in: Handb. Electrochem., Elsevier, 2007: pp. 391-428. doi:10.1016/B978-044451958-0.50023-9.

[16] X.J. Huang, A.M. O'Mahony, R.G. Compton, Microelectrode arrays for electrochemistry: Approaches to fabrication, Small. 5 (2009) 776-788. doi:10.1002/smll.200801593.

[17] J.J. Hickman, D. Ofer, P.E. Laibinis, G.M. Whitesides, M.S. Wrighton, Molecular self-assembly of twoterminal, voltammetric microsensors with internal references., Science. 252 (1991) 688-91. doi:10.1126/science.252.5006.688.

[18] N.L. Abbott, D.R. Rolison, G.M. Whitesides, Combining Micromachining and Molecular Self-Assembly To Fabricate Microelectrodes, Langmuir. 10 (1994) 2672-2682. doi:10.1021/la00020a028.

[19] J.P. Metters, C.E. Banks, A. Escarpa, M.C. González, M.Á. López, Microelectrode Designs, in: Agric. Food Electroanal., John Wiley \& Sons, Ltd, Chichester, UK, 2015: pp. 137-168. doi:10.1002/9781118684030.ch6.

[20] A.N. Bezbaruah, T.C. Zhang, Fabrication of anodically electrodeposited iridium oxide film pH microelectrodes for microenvironmental studies, Anal. Chem. 74 (2002) 5726-5733. doi:10.1021/ac020326l.

[21] M.H. Wang, B.W. Ji, X.W. Gu, H.C. Tian, X.Y. Kang, B. Yang, X.L. Wang, X. Chen, C.Y. Li, J.Q. Liu, Direct electrodeposition of Graphene enhanced conductive polymer on microelectrode for biosensing application, Biosens. Bioelectron. 99 (2018) 99-107. doi:10.1016/j.bios.2017.07.030.

[22] Hybridization of two different strands of DNA or RNA / mfold.rit.albany.edu, (n.d.). http://unafold.rna.albany.edu/?q=DINAMelt/Hybrid2.

[23] T.J. Davies, R.G. Compton, The cyclic and linear sweep voltammetry of regular and random arrays of microdisc electrodes: Theory, J. Electroanal. Chem. 585 (2005) 63-82. doi:10.1016/j.jelechem.2005.07.022. 
[24] Y. Du, S. Guo, S. Dong, E. Wang, An integrated sensing system for detection of DNA using new parallelmotif DNA triplex system and graphene-mesoporous silica-gold nanoparticle hybrids, Biomaterials. 32 (2011) 8584-8592. doi:10.1016/j.biomaterials.2011.07.091.

[25] D.A.J. Rand, R. Woods, The nature of adsorbed oxygen on rhodium, palladium and gold electrodes, J. Electroanal. Chem. 31 (1971) 29-38. doi:10.1016/S0022-0728(71)80039-6.

[26] M. Ishigami, J.H. Chen, W.G. Cullen, M.S. Fuhrer, E.D. Williams, Atomic structure of graphene on SiO 2, Nano Lett. 7 (2007) 1643-1648. doi:10.1021/nl070613a.

[27] F. Li, Y. Huang, Q. Yang, Z. Zhong, D. Li, L. Wang, S. Song, C. Fan, A graphene-enhanced molecular beacon for homogeneous DNA detection, Nanoscale. 2 (2010) 1021. doi:10.1039/b9nr00401g. 